\title{
五十年來的相对論学說
}

\section{L. 英費尔德}

（波闌科学院院士）

今年是从古以來最偉大的科学家之一, 阿尔 柏特 - 爱因斯坦, 逝坦的一年。今年是他的偉大 的工作，相对諭，産生的第五十週年。这二个同 時來到的事件, 將我們的注意力集中到他在人類 思想史上的独特的成就上去。

今天的物理学家把相对論学說看作經典的学 說, 相对論爭論的時代似乎久已过去了。这种看 法对於狭义相对論学說是正確的, 但是对於展义 相对論学說倒不見得是十分正確的。在这裏我要 从 1921 年劳埃敉授發表的“廣义相对論”一書的 序言中引用兩句話:

“今天相对論学說一方面受到非常多的称賀， 一方面受到頗多的資难。在这兩方面叫得最响的 人們有一點是共同的: 他們根本不理解他們所談 的东西! ”

今天这兩够話的正確性远不如 1921 年的時 代。但是它們仍然是正確的，因为虽然我們在物 理学家中已很难找到反对陝义相对論的人，然而 仍还有些物理学家是不承認廣义相对論的。有些 区对爱因斯坦的廣义相对諭的物理学家傾向於其 它的引力学說; 另外有些人要改变引力学說的解 釋。

从物理学的覌點看來, 涨义相对論確实要比 廣义相对論重要得多; 但是当我們考虑到人颣智 慧的成就時，廣义相对論就又比狄义相对論颖得 重要了。

这寡我想引用一下爱因斯坦同我閣於这个論 題的一段談話。在普林斯顿我有一次对爱因 斯坦說: “即使你沒有發現狹义相对諭, 我相 信一一不会在太久之後一一狄义相对論也会被人

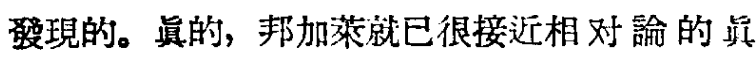
理。”爱因斯坦回答說: “是的, 这是正確的, 但
只是对於狭义相对論是正確的; 对於廣义相对諭 並不正確。我怀疑如果我沼有郋現廣义相对諭， 人們今天是否能知道它。”

度义相对論是从陝义相对論座生出來的。落 是我們願意（就像我們所做的那样）檢查相对論 的腾展史, 那密我們在廣义相对論上要比在狄义 相对論上花費更多時間。因为我們很难祝在㢸义 相对論中有什照持物是不为每一个理諭物理学家 甚至实驗物理学家所了解的。而在另一方面, 知 道廣义相对論的人却少得多。一个不大獲得廣义 相对論的人仍可以是一个物理学家。有些物理学 家認为廝义相对論泜是形式的东西, 而且同实驗 联系極少。認为三个論証——就像葉些物理学家 所主張的一一都是可疑的, 而且和它們有關的事 实都是索然味的。

也有些物理学家認为这种先入之見的刵断是 錯誤的; 認为廣义相对論解决了引力問題; 認为 廣义相对論是非線性場諭中的一个奇妙的例子; 認为它对於物理学其它各部門的影响將会愈來愈 大。我就是这些物理学家中的一个, 但是我不相 信廣义相对論对於我們理解基本粒子的構造会有 很大的帮助。

說明白了这些以後, 現在我就開始談談相对 論歷史梫展的本身。我的報告是不詳尽的也是不 客覌的。主題本身不旮詐客覌，報告時間的限制 也不允許我作詳尽的論述。

1905 年出版的第 17 姿“物理学年報” 內有一 篇爱因斯坦的 30 頁的喻文: “論运動物体的电 動力学。”論文的題目似乎很平裳, 可是当我們 讀它封，我們幾乎主刻会注意到它是和其它諭交

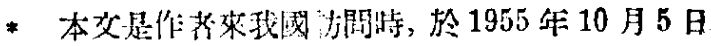
向北京科学工作者源作的鼣告。
. 76 .
科学通報
1955 年 


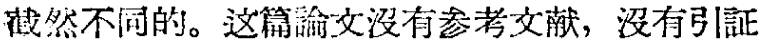
权威，只有很少解釋性的附註。交休簡明，交章 大部分不要高深的物理学知識都可以讀得懂。如 果說有一个㝛閲老的話, 人們会覚得奇怪, 这样 一䈐和通常的淪文迥然不同的諭文, 怎样会为審 閱者所通过。更特別的是, 望充分理解这篇論文

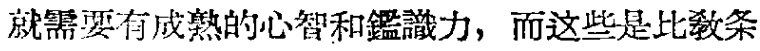
的知識这为稀侍和更为珍貴的。論文的寫法与文 体, 就在今天看來，也还是十分新鮮的。它仍然 是学替相对諭的最仔的資料之一。50 年前, 当 時著者才 26 瘧; 在瑞士伯尔尼的專利局 当一名 躘䝿。

在爱因斯坦論文的第二節中，我們讀到:

1 如果有兩个坐标系, 作相对等速运動, 那 末物理系繶在改变狀态時所遵循的各种規律無論 对这兩个坐标系的那一个來涗, 都是一样的。

2. 不管光線是从固定的或是从运動的光源㻐 射出來的, 每一光線在一个静止坐标系中總是以 固定的速度 C 运動着。

这是兩个假定: 伽利略的相对性原理的假定 和光速不变的假定。大家都知道罗倫茲变換可以 从这兩个假定推纯。它們是狭义相对論的基礎。 它們也是引起我們对空間時間覌念作根本修正的 猳設。

在下一然“物理学年報”中有一篇 題 名为: “物体的慣性是否同它的能量有關? ”的短文。若 翨我份这篇文章中的覌念是震動世界的，也不算 誇大, 因为在这赛我們第一次找出一个新現象的 理論基礎, 而这新現像为科学和技術開闢了無限 的远景。这篇文章說: 在原理_上, 原子能的利用 是可能的。 40 年後, 許多科学家的研究 証 明 原 子能用於战爭是实际可行的。这个証明很完全, 正如廣峋和長崎受到了完全的毁隇一样地完全, 在那裹 29 万男女會被突然殺害。現在在这个原 理提出 50 年後的今天, 終於又証明了原子能地 可以用於人類的置利事業。原子能的兩种应用的 种子同是由世界上一位最和平的、痛恨暴行的、 卑䂓野蛮力量的孤独的人种下的，这是一个痛苦 的諷刺。原子能的毁減力量首先在兩座城市的試

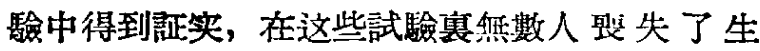
命，而一直到十年以後，当苏联建立起第一座小 等的原子能㥅电故時，才開始了原子能的和平利
用, 这同样是一个痛苦的諷刺。

爱因斯坦这篇短交的結論說:

“物体的質量是它的能 量 的量度。若是它的 能量变化 $\mathrm{L}$, 那涞它的質量就变化 $\mathrm{L} / 9 \cdot 10^{20}$, 这 赛能量以厄格計，質量以克計。

“有些物体內所含的能量可能有很大的变化 (例如鐳監)，那未關於这个理論的行試驗是否能成 功呢?”

对这篇論交的反应怎样? 最初幾 乎沒有 反 应。在今天, 人們或培能更快地承認一些重要的 研究結果, 而且一篇革命性的新論文常常会引起 其他更多的人來把新思想更細緻地加以補充，並 在数学上加以發揮。可是在我們这一世紀的科学 史中世曾有过相反的例子: 例如在德布罗意的論 文弡表後的兩年間, 就沒有發表过一篇有關这个 題目的文章。对於爱因斯坦的著作也有類似的情 况。大約 4 年後, 即 1909 年前後才有大 量諭进 相对論的論文出現，物理学家們的確經过一段頗 長的時間才認識一些最重要的諭文。

可是我知道在这期間也有一些物理学家睤經 細心閱讀爱因斯坦的論文，並且在論文中看到新 科学的誕生。我的朋友罗力亞教投（波闌人）曾 告訴我他的先生魏德柯夫斯基呚授一一位受过 很好的数育和在物理学上具有很高見地的物理学 家一一筧怎样閱讀爱因斯坦的論交和他怎样兴奋 地对罗力亞說: “快去讀爱因斯坦的論 文。一位 新的哥白尼降生了。”

直到 1909 年,很多很多科学家的注意力才被 爱因斯坦的研究成果所吸引。使相对諭被更多的 人們知道的一个因素是 1908 年閔可夫斯基的講 演: “空間和時間”。他在第 18 屆科学家及博士协 会会議上所作的这个著名的講演，我想大概是他 最後的一次公開講演。不久以後，他就天亡了。䦡 可夫斯基講演的头幾句話是对於爱因斯坦覌點在 現代思想中的哚刻影响的預言性的說明，他說:

“各位先生: 我想同各位談的空間和時 間 的 覌點是建築在物理学实驗的基礎上的。这些覌點 的力量就在於此。它們的傾向是根本性的。从現 在起, 空間本身和時間本身要下降到 影子的地 位, 只有兩者的統一才能保持它的独立性。” 閔可夫斯基的數学天才使得爱因斯坦的观點 得到了新的幾何形式，从而充分顯示了爱因斯坦 
的覌點的美麗和养素。閔可夫斯基的著作公佈以 後, 我們知道了一切自然規律都能寫成矢量或張 量形式，这些矢量和張量都是四維時空流形中的 天量和張量。進一步的發展又在矢量和張量之外 加上了旋量。

从歷史的角度看來, 涨义相对論的進一步郋 展是同廣义相对論的發展相联系的。但由於我們 留在这軎把兩种相对諭分開來榉，所以我只再稍

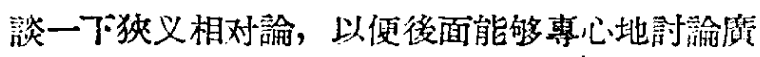
义相对論。

陝义相对諭以後的發展是一段它在我們知傤 領域中勝利前淮的歷史。是的, 我只要举出三个 稗煌地証实弞义相对論的現像：質量对於速度的 關联性，埃夫士所作關於运動的鐘的快慢的精叮 实驗，介子寿命对於介子速度的閏联性。这幾个 实驗同其它許多实驗一样，都是为狹义相对諭作 讋讙的，汥有一个实驗是反对它的。

我們世紀內在現象預兒方面的兩个最愇大勝 利的歴史是同狭义相对諭的歷史密切联系的。我 这是指德布罗意波和陽电子理諭。德布罗意波的 存在的预見与罗倫茲变換有泌切閔係, 而陽电子 理諭同相对論形式的狄拉克电子方程式有泟密切 阖係。

在这哃我还要提起另外一个現象, 这个現像 的解釋很可能你們还不知道，因为这篇諭文現在 才付印。一位年輕的波蘭物理学家魏尔勤指出从 核子运動方程式的相对論形式出發，可以得出核 子从一个力中心所受到的乕力, 䣰要核子同力中 心十分接近，並且假定介子場是标量場或胳标量 場，而不是矢量場。这是純粹相对論的效应。因 此, 藉助於相对諭, 似乎可从介子場的場方程式 中求得雅斯特洛夫和勒未的假設。

現在我們做開狹义相对論，來談廣义相对 論。

爱因斯坦曾告訴我祬，他从幼年起就想过下 面兩个閣題:

1. 若是有人正在跟着光線跑並努力去赶上 它, 將会弡生什麼?

2. 把一个人關在一个自由下落的昇降机上, 將会弡生什麼?

从头一个問題的答案腾展了狄义相对論, 从 後一个的答案弡展了廣义相对論。
後來由狄义相对渝所解决的矛盾是当時物理 学家們都知道的。浙㭙産生狹义相对諭的条件已

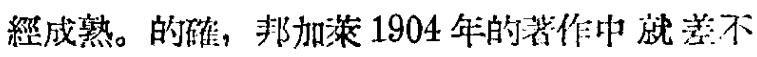

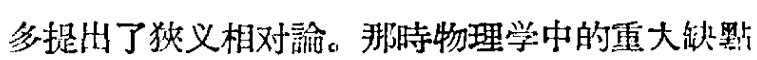

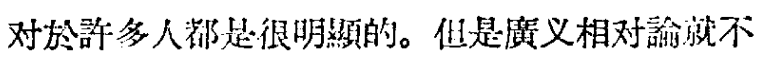
是这样。爱因斯坦是在这打面罗到图难和牙质而

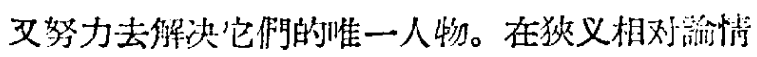

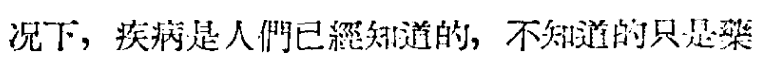

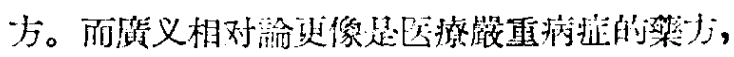

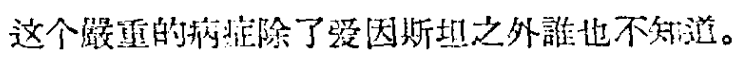

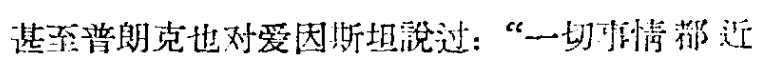

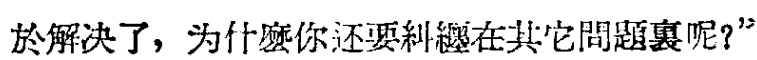

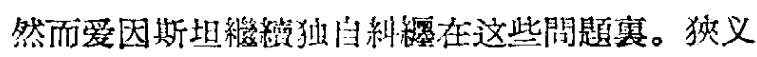
相对諭和廣义相对論之間相隔着 8 年的時㯕; 8 年的經学思考終於社引力这个重大問題上策生了 新的解答。

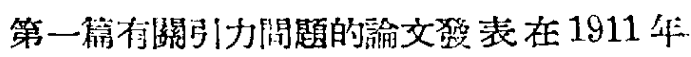
的“物理学年報”上，撚名为“引力对於光的傅提 的影响”。这是一篇極有兴趣的馀文, 因为它比

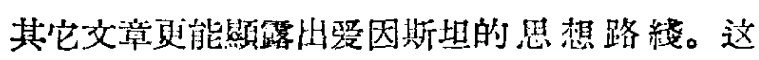
䈐論文有一部分是錯言吴的: 它包含了牛黃理、猜 想, 並且戲剧性地澺譏到员理已經不远, 虽然它 包含的是很不相同的东西。它質示了黑喑中透露 出來的第一道微弱的光綫。它反肤了爱因斯坦对 於理想实驗的爱好和他的孩子似的怀疑簡單事物 的能力; 事情是这样地簡單而又司空見慣, 所以 别人一直不自注意过它。

自从咖利略以來, 物理学家就烟道所们的到。 体都以相同的加速度降落。除了爱因斯坦以外， 沒有人再对这个規律感到警奇。实驗在我們能佔

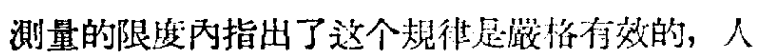
們以为这一事实-劳永逸地解决了問題。

数育使人們失去怀疑的能力。只有天少少能

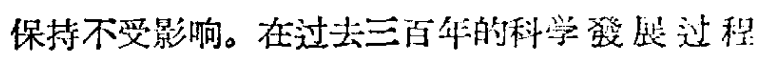
中，爱因斯坦是把加逝度的相同存作重瑟术彻線

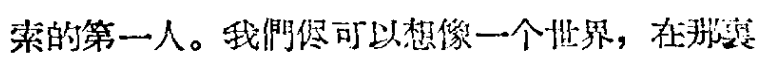
加速度相同的規律是繁效的; 在那个世界重, 大

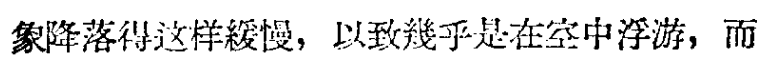
小孩却以危險的極大的加速沾降落到地面上來。 可是我們地球的引力㳟却使小孩与大象都以同一 加速度下降。这个重哲線奖的等义是什麼? 在古 典力学体系裹, 它似哲只是个偶然事件罢了! 
正如我在前边提过的, 爱因斯坦在兒童時代 所想到的一个閣在自由降落的舁降机裹的人的景 象, 終於在很多年以後把他引倒廣义相对諭的概 念。在現在我們所說到的爱因斯坦的諭文裹, 线 們也看到了一一隱約地一一自由降落的昇降机裹 一位观察者的照象。这堡, 从这样一个理想实 驗出资, 爱因斯坦得出了引力場影响下光綫登生 弯曲的結諭! 这个計算並不十分正確。爱因斯坦 引用的數字結果太小了。他还沒有展义相对諭的 充分知識。在以後的 4 年裏他又重新進行計算並 且作了修正，在这期間方才立分䓵得馓义相对論

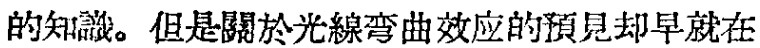
1911年爱团斯坦的論交中出現。他以以下藷名的 話結束那篇論文:

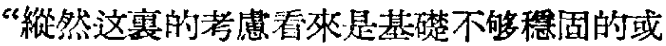
甚至是冒險的，但是我迫切地朌望天文学家們關 心这个問題。先不要管是什染理論，一个人必須 問自己，用我們今天所有的方法是否能够猡引 力場对於光的傳播的影响。”

爱因斯坦向天交学家們的挑战 產 生了什麼 呢? 8 年过去了。这期間爱因斯坦从杪拉格要居 到苏黎支，又从苏黎支要移到柏林。在非琵他逢 到了第一次世界大战，並且在朋赛完成了他的廣 义相对論的論文。

廣义相对諭的知識非常䌊慢地从理論物理学 家傅播到实驗物理学家、天文学家、數学家和哲 学家。人們都把它看作一个極度困难的論題。我

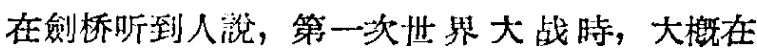
1917 年，阿瑟・爱丁頓磿士會作过關 於 廣义相

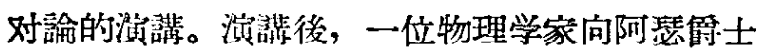
說: “这是一篇動人的演满。你是懂得相对論的 三个人中的一位。”当時爱丁頓脸上現出了苦惱的 表情, 这位物理学家就說: “爱丁頓教授不要感賞

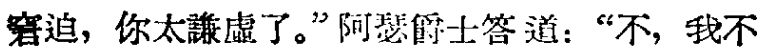
悉迫，我只是怀疑誰是第三个了解相对論的人。” 那時候了解廣义相对諭所必需的數学知識还 不为很多人所知道，而且也沼有得到充分的發

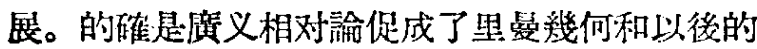
非里曼线何的進一步發展。相对論学說大大地刺 激了这些數学部門的成長。

大战以後，廣义相对論的知識傳播到英國、 苏联和其他國家。1919年英國派出了兩个科学工
作榢，一榢到巴西的苏布拉尔，一隊到非洲海岸的

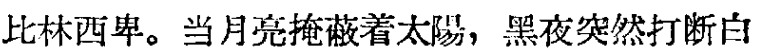
畫時, 科学家們拍攝了太陽周葍的可見的星体, 目的是找出光綫弯曲效应在數量上是否闰度义相 对諭的預料一致。当時旂宣仿观測結果似我光䊅 地証实了爱因斯坦的预見。虽然後來的測量稍稍 破坏了这一致性，但是今天对於引力場中光綫的 轉弯已經是汥有什麼可怀疑了。

光綫的弯曲現象吸引住了整个文朋奾桇的想 像力。1920 年以後, 爱因斯吅这位垃界上最洋 虚的人很快成了一位最有名的人。我相信爱因炘 坦的名望突然昇高的原因，在於人類对和平的渴 壁。这裹是这样一个自然界的現像，如同日蝕時 天空知出装星的現象一样奇異和不可思議; 这也 是这样一个自然界的現象，它的理論是由一位 德國教授提出的，而英國科学家們証实了这个理 諭。在这裹，兩年前互相作战的兩个國傢的科学: 家進行了合作。我相信这也是为什染反動势力反 对爱因斯坦的原因。他成了世界上最著名的科学 家，同時也是最受贊美的和最受嘲笑的科学家。

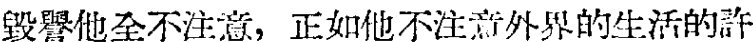

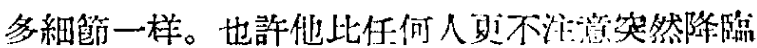
的声譽。

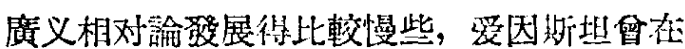

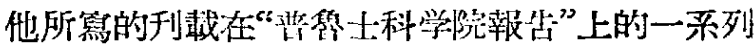
諭交裏一次再一次地捉出刑修改了成义相对諭。 他不止一次地不得不回头检查自己的各个工作步 聚，剔除錯望，这才愈來愈深入地認識了引力問 題。大約在 1916 年，廣义相对論的理諭体系基 本上芫成了, 整个理論再度被竄在一篇總結性的 文章寒，弡表在“物理学年報”上，題为“廣义相 对諭的基礎。”这以後就沒有再作过根本的改变, 虽然加源了許多新的結果, 並且理淪上也進一步 有了發展。

廣义相对論所得到的不同於古典力学的結論 中，有一个是十分著名的：水星近日點的進動。 它在 1916 年成为相对論理論的第一个证据。这 个結論是当封著名天文学家許瓦茨希尔德从爱因 斯坦的方程式嚴格地推断出來的。可是这样說法 在某媵點上是太过於簡單化了。这个問題的全部 歴史裂複雜得多，我簡單地報告一下。

1916 年左右提出來的廣义相对諭焉築在 能 
个支柱上面。一个是場的方程式, 即描述引力 或幾何場在空間時間中的变動的方程式。另一个 是运動方程式, 它告訴我門一个質點怎样在引力 場中运動。这些方程式代替了引力正此於加速度 的萑的牛頓运動方程式。而且現在在監义相 对 論中, 运動方程式正和其它自然規律一样, 不僅 在慣性坐标系中有效, 而且在任意坐标系中地都 是有效的。

因此，若是我們想知道行星在太陽引力場中 的运動規律, 那末我們首先必須根据廣义相对䜽 的場方程式求出太陽的引力場。然後我們必須在 已知的場中应用运動方程式, 从而求出行星的运 動。这恰恰是許瓦茨希尔德运用高度熟練的數学 技巧所作的。但是他的結果只在行星比太陽小得 多的情况下才是有效的。在双星的情况下怎样 呢? 一一那就是馀, 不是一体問題而是兩体問題 的情况下怎样呢? 这裏我們不能使用許瓦茨希尔 德的方法。这裹廣义相对諭的第二个支柱是無效 的。

我們虽然有場的方程式和运動方程式, 但是 在廣义相对諭裹可以明顯地看出，运動方程式的 有效性是受限制的。1938 年以前我們一直不知 道怎样在廣义相对諭中解决双星运動問题, 虽然 在古典力学中这問題的解决幾乎是無關輕重的。 在古典力学中, 我們很难說双星問題比一个重的 太陽加上一个小的行星的問題更加困难。

爱因斯坦曾有很長一段時間相信廣义相对論 中的运動方程式是不必要的, 他認为不必去假定 这种方程式, 認为它們可以从場方程式推濒出 來, 認为我們可以取消並忘却运動方程式, 認为 座义相对諭的唯一支柱就是場的方程式。这証明 是正確的, 但要花費很長的時間來証明。在1916 年左右, 所有的技巧方法都巨掌握在數学家和物 理学家的手裹。他們都知道場的方程式。所需要 作的就是証明这方程式裹包含着运動方程式。这 好像是去發掘我們已知道埋在哪寒的地下宝物一 样。爱因斯坦會暦次地企圖解决这个問題, 文屡 次地倣開它並轉向其它問題, 然後又雷回头來研 究这个問題。在这時侯希特勒上台了，1933 年 爱因斯坦离開了德國, 到普林斯頓居住, 並克任 高級研究院的枚接。1938 年表的著作，第一次 从廣义相对論的場方程式中推演出來了双攵运動
的固有方程式，它比牛頓方程式逐为精確一步。

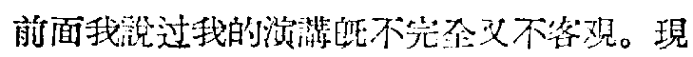
在为了嚴整起兒, 现想再捉一下谁面所略去的 一些重要論題，它們是：第三个炇应(蚛紅向移

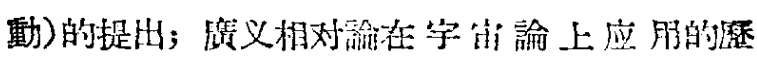
史; 一元理諭的探討，在这个䦌題上爱因斯坦尃 心研究了 35 年, 而且到卧死前他还在繼繥研究

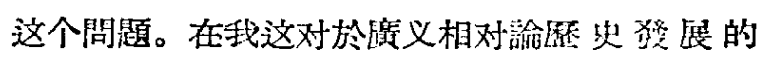
簡短回顧中，我所談到的問題当然都是主烷地选 擇的。

还有一个問題也是我想回答的, 就是物理学 界对於廣义相对論有些什㦄看法。

我相信大多数物理学家都会承認这是唯一合 理的引力場理論, 而且是一个很美麗的学說。其 它的理論也是存在竞的，但是爱因断顷的理渝由 於它在數学上的美酶，罯輯上的簡單以及它与实

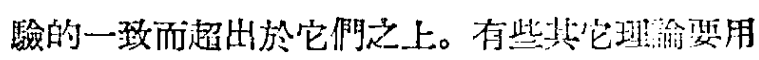
一个特殊常數才能得出水星近日點的進動; 可 是爱因斯弨的学得一一这是它的極大优點——却 用不着任何新的常數。別的引力理諭大多數假定 狄义相对諭中的慣性参将系統的存在，在有些理 諭裹, 甚至双星的質量师心还有加速度! 可是另 一方面, 爱因斯坦的展义相对部却是很合理的: 即双星的質量中心具有约速。在那些拥護不同於 爱因斯迎理論的引力理諭的物理学家湖康來, 廣 义相对論是太偏激了! 但是我相信它的力量恰恰

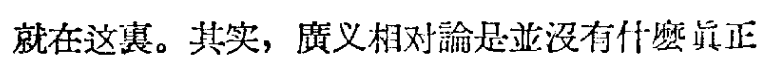
的勁敌的。廣义相对諭学镜已有 40 年的虂史。 同它差不多同時提出的理耳学說在 12 年 以後就 已被薛定諤和游森堡的学說所代替了。然而可注 意的是 40 年以後爱因斯坦的学說依然是富 有生 命的学說。閣於这个学訜还繼續有重要諭文出 現, 不过它已不是今天物理学家的中心兴趣所在 而已。

正如我所說过的, 由於各种理由, 有少數物 理学家寧願接受其它的一些引力学說。然而也还 有一大部分物理学者, 杂接受度义相对而的數学 結構的同時，却又不同意爱因斯坦对基本、覌念的 解釋。

廣义相对論的兩个正要思想是:

1.引力場和度規場是等同的;

[下接 43 頁] 